\title{
Editorial: Adaptive Immunity in Pregnancy
}

\author{
Marie-Pierre Piccinni ${ }^{1 *}$, Sarah Anne Robertson ${ }^{2}$ and Shigeru Saito ${ }^{3}$ \\ ${ }^{1}$ Department of Experimental and Clinical Medicine- University of Florence, Florence, Italy, ${ }^{2}$ Adelaide Medical School and \\ The Robinson Research Institute, University of Adelaide, Adelaide, SA, Australia, ${ }^{3}$ Department of Obstetrics and Gynecology, \\ University of Toyama, Toyama, Japan
}

Keywords: T helper cells, T reg cells, placenta, spontaneous abortion, pregnancy, maternal-fetal immune tolerance

\section{Editorial on the Research Topic}

\section{Adaptive Immunity in Pregnancy}

One of the most remarkable features of reproductive biology is the fact that a healthy woman can successfully carry her genetically disparate conceptus to full term, without immune rejection.

The juxtaposition of the placenta and decidua creates what is referred to as the 'fetal-maternal interface', where placental trophoblasts of fetal origin and maternal uterine lymphocytes come into close contact. Due to the presence of paternal class I HLA-C molecules on trophoblasts, the conceptus can be considered to resemble a semi-allograft. Conceptus-derived and placental-derived antigens act to both prime maternal $\mathrm{T}$ cells and render the conceptus potentially susceptible to inflammatory effector activity or T cell-mediated attack. After presentation of paternal alloantigens by maternal antigen presenting cells (APCs), the maternal alloantigen-specific T cells proliferate and secrete cytokines, responsible for the activation of allograft rejection or tolerance mechanisms, respectively promoting pregnancy failure or fetal survival.

Therefore, the quality and strength of the adaptive immune response is critical to healthy

Edited and reviewed by: Herman Waldmann, University of Oxford, United Kingdom

*Correspondence:

Marie-Pierre Piccinni mppiccinni@hotmail.com

Specialty section:

This article was submitted to Immunological Tolerance and Regulation,

a section of the journal

Frontiers in Immunology

Received: 03 September 2021 Accepted: 20 September 2021 Published: 04 October 2021

Citation:

Piccinni M-P, Robertson SA and Saito S (2021) Editorial: Adaptive Immunity in Pregnancy.

Front. Immunol. 12:770242. doi: 10.3389/fimmu.2021.770242 pregnancy. There is accumulating information that imbalance in the numbers, phenotypes and functional activity of $\mathrm{T}$ cell subsets can adversely impact fertility and pregnancy health. Predominant Th1, Th17 and Th17/Th1 immunity and decreased Th2, Th17/Th2 and Treg cells are associated with recurrent pregnancy loss (RPL) of fetuses with normal fetal chromosomal content. Various subsets of $\mathrm{T}$ cells are essential for pregnancy tolerance and interact in networks with innate immune cells to counteract inflammation and promote robust placental development. In fact, immune cells that populate the decidua are specialized not only to minimize events that might evoke conceptus attack, but also to foster placental development and function and to combat infections during pregnancy.

In addition, $\mathrm{T}$ cells are commonly perturbed in late gestation disorders including preeclampsia, fetal growth restriction and spontaneous preterm birth. There is some evidence that $\mathrm{T}$ cell disturbances precede the onset of symptoms and contribute to disease pathophysiology through events around the time of implantation and early placental development.

In this Research Topic we welcomed six original articles and four review articles, which discuss the role of novel immunosuppressive cells and molecules regulating fetal tolerance and development.

During pregnancy, sex steroid hormones like estrogen, progesterone, hCG but also a progesterone-induced mediator, the progesterone-induced blocking factor (PIBF), which conveys some of the immunological effects of progesterone, suppress effector immune activation resulting in successful pregnancy. Csabai et al. reports that the implantation rate is decreased in mice treated with anti-PIBF antibody. In these anti-PIBF-treated mice, NK activity, IL-12A mRNA expression in 
$\mathrm{CD}^{+} \mathrm{T}$ cells and Th1 differentiation are increased, whereas the expression of mRNA for IL-4 (a Th2-type cytokine) is decreased in $\mathrm{CD}^{+}{ }^{+} \mathrm{T}$ cells (Figure 1). Thus, PIBF plays an important role in implantation by upregulating a Th2-type and downregulating Th1-type immune responses.

As well as hormones, other molecules including the prostaglandins (PG) can also regulate immune cells in pregnancy. In particular Andrade et al. show that $\mathrm{PGE}_{2}$ also contributes to immune tolerance, by inhibiting platelet aggregation and IL-5 and IL-13 production by innate lymphoid cells (ILCs), and by suppressing neutrophil, NK cell and monocyte effector functions (Figure 1).

The role of Treg cells in pregnancy is extensively developed in the Research Topic. Huang et al. reviewed the role of Treg in normal pregnancy, in implantation failure, miscarriage, endometriosis and preeclampsia. Krop et al. described not only the role of well-known Foxp $3^{+}$Treg cells in pregnancy, and also importantly the role of the lesser-known Foxp $3^{-}$Treg cells, which include HLA-G Treg cells, Tr1 cells that secrete IL-10 and TGF- $\beta$, Th3 cells that secrete TGF- $\beta$, IL-10, and IL- $4, \mathrm{CD} 8^{+}$ Treg cells, NO-Treg cells, TIGIT $^{+}$Treg cells, and $V \delta 1^{+} \gamma \delta \mathrm{T}$ cells (Figure 1). However, the relationship between these Foxp3- Treg cells and pregnancy disorders remains to be clarified.

Morita et al. examine $\mathrm{CD}^{+} \mathrm{T}$ cells that are less well studied in pregnancy, in particular clonally expanded $\mathrm{CD} 8^{+} \mathrm{T}$ cells, using single cell analysis of $\mathrm{T}$ cell receptor $\beta$ (TCR $\beta$ ) sequences. Clonally expanded $\mathrm{CD} 8^{+} \mathrm{T}$ cells may be a surrogate marker for fetal/paternal antigen-specific $\mathrm{CD}^{+} \mathrm{T}$ cells. The authors show that clonal $\mathrm{CD} 8^{+} \mathrm{T}$ cells are more abundant in effector memory $\mathrm{CD}^{+} \mathrm{T}$ cells $\left(\mathrm{CD} 8^{+} \mathrm{EM}\right)$ and that there are more $\mathrm{CD} 8^{+} \mathrm{EM}$ cells in the decidua than in the peripheral blood. The clonal $\mathrm{CD}^{+} \mathrm{T}$ cells increase from early to late pregnancy, and PD-1 expression, which suppresses cytotoxic activity, is low on clonal CD ${ }^{+} \mathrm{T}$ cells in early pregnancy but high in late pregnancy. The clonal PD1 $\mathrm{CD}^{+} \mathrm{T}$ cells increase in miscarriages with normal fetal

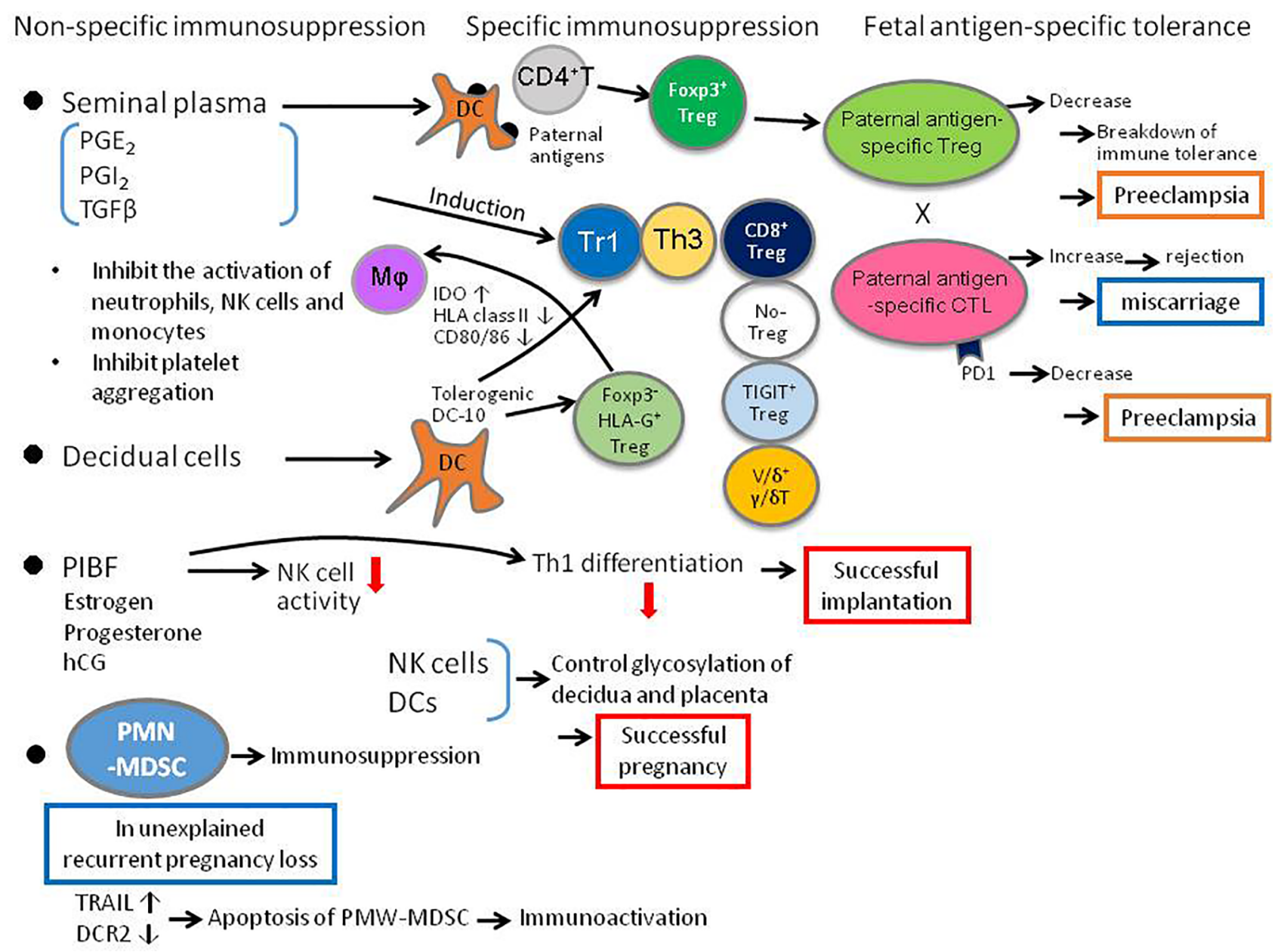

FIGURE 1 | Diagrammatic summary of critical elements of the adaptive immune response to pregnancy, that determine the balance between T cell tolerance and T effector function, and influence the outcome of pregnancy and infant health. DC, dendritic cell; IDO, indoleamine-dioxygenase; M $\Phi$, macrophage, PD1, programmed death 1; PIBF, pregnancy-induced blocking factor; PMN-MDSC, polymorphonuclear myeloid-derived suppressor cell; PGE, prostaglandin E2; PGI, prostaglandin I: Tr1, T regulatory 1; Th, T helper; TIGIT, T cell immunoglobulin and ITIM domain; Treg, regulatory T cell; uNK, uterine natural killer cell. 
karyotype (Figure 1). The percentage of clonal $\mathrm{CD}^{+} \mathrm{T}$ cells is not different in preeclampsia compared to normal pregnancy, but PD-1 expression is significantly decreased, suggesting an increased cytotoxic activity against fetal antigens in preeclampsia.

Immune cells can not only act by inducing trophoblast tolerance but can also affect the structure of trophoblast by altering the glycan chains of trophoblast. Dendritic cell activity is particularly important, and may be amplified if NK cells are removed as demonstrated by Borowski et al. Thus, immune cells can influence the placental glycade and could impact placental and fetal development (Figure 1). The relationship between the alteration of trophoblast glycan chains and immune cells in miscarriage and preeclampsia is an interesting topic for future clarification.

Non-immune cells can also regulate immune cells responsible for immune tolerance in pregnancy. Gori et al. showed that endometrial decidualization increases tolerogenic dendritic cells named DC-10 cells, which secrete IL-10 and induce different regulatory $\mathrm{T}$ cells (Treg cells), including $\mathrm{HLA}^{-\mathrm{G}^{+}}$Treg cells, $\operatorname{Tr} 1$ cells and Th3 cells (Figure 1).

Recently, polymorphonuclear myeloid-derived suppressor cells (PMN-MDSCs) have been found to increase in the pregnant uterus and play an important role in maintaining pregnancy. Li et al. show that the number of decidual PMNMDSCs decrease in patients with unexplained recurrent pregnancy loss. PMN-MDSC apoptosis, increased by elevated TRAIL and decreased by DcR2, could explain the decreased number of PMN-MDSCs in unexplained recurrent pregnancy loss (Figure 1). The interaction between PMN-MDSCs and immune cells such as Treg cells, need to be investigated.

Van der Zwan et al. used mass cytometry to analyze lymphocyte subpopulations in the decidua and peripheral blood. Such analysis could be helpful for the classification of immunocompetent cells and to clarify the role of each of these cells in the decidua. This promising technology, which may serve as a foundation for further identification of immune subsets in healthy and complicated pregnancy, is set to offer further advances in the future.

Finally, it is important to recognize that the maternal adaptive immune response to pregnancy has consequences not only for pregnancy outcome, but also for the health of the child after birth. Albrecht et al. review emerging studies showing that cellular immunity is transferred from mother to child not only through IgG transfer during pregnancy, but also by maternal cellular immunity transmitted to the child through lactation after birth. This article introduces the interesting possibility of transferring immunity to the fetus by vaccination during pregnancy.

In summary, this collection of papers provide a snapshot of the state of this field and provide new insight on the mechanisms and significance of the adaptive immune response to maternal and infant health. Collectively the work highlights the imperative to further delineate the underlying mechanisms by which maternal tolerance is generated and mediated, so that interventions to protect against immune-based pregnancy disorders arising from compromise maternal tolerance can be advanced.

\section{AUTHOR CONTRIBUTIONS}

All authors listed have made a substantial, direct, and intellectual contribution to the work and approved it for publication.

\section{ACKNOWLEDGMENTS}

We thank the authors of the 10 publications of the Research Topic for their high-quality work. We thank the Frontiers in Immunology Editorial Office and the Editor for their support.

Conflict of Interest: The authors declare that the research was conducted in the absence of any commercial or financial relationships that could be construed as a potential conflict of interest.

Publisher's Note: All claims expressed in this article are solely those of the authors and do not necessarily represent those of their affiliated organizations, or those of the publisher, the editors and the reviewers. Any product that may be evaluated in this article, or claim that may be made by its manufacturer, is not guaranteed or endorsed by the publisher.

Copyright (c) 2021 Piccinni, Robertson and Saito. This is an open-access article distributed under the terms of the Creative Commons Attribution License (CC BY). The use, distribution or reproduction in other forums is permitted, provided the original author(s) and the copyright owner(s) are credited and that the original publication in this journal is cited, in accordance with accepted academic practice. No use, distribution or reproduction is permitted which does not comply with these terms. 